\title{
ADH4 Gene Variation is Associated with Alcohol Dependence and Drug Dependence in European Americans: Results from HWD Tests and Case-Control Association Studies
}

\author{
Xingguang Luo ${ }^{1,2}$, Henry R Kranzler ${ }^{3}$, Lingjun Zuo', ${ }^{1,2}$ Jaakko Lappalainen ${ }^{1,2}$, Bao-zhu Yang ${ }^{1,2}$ \\ and Joel Gelernter*, 1,2 \\ 'Department of Psychiatry, Yale University School of Medicine, New Haven, CT, USA; ' VA Connecticut Healthcare System, West Haven Campus, \\ CT, USA; ${ }^{3}$ Department of Psychiatry, Alcohol Research Center, University of Connecticut School of Medicine, Farmington, CT, USA
}

\begin{abstract}
The alcohol dehydrogenase $(\mathrm{ADH})$ family constitutes one of the key sets of enzymes responsible for the oxidation of alcohol. The $\mathrm{ADH} 4$ gene, an important member of this family, is a functional and positional candidate for alcohol dependence. The present study aimed to investigate the relationship between $\mathrm{ADH} 4$ gene variation and alcohol dependence and drug dependence in EuropeanAmericans (EAs) and African-Americans (AAs). Seven single nucleotide polymorphisms (SNPs) spanning the ADH4 gene were genotyped in 365 healthy controls ( 317 EAs and 48 AAs) and 561 subjects (400 EAs and I6I AAs) affected with alcohol dependence and/or drug dependence (436 with alcohol dependence; 356 with drug dependence). Hardy-Weinberg equilibrium (HWE) for the genotype frequency distributions of these markers was tested in all phenotype groups to evaluate association between ADH4 gene variation and phenotypes and to fine-map the disease risk locus. The allele, genotype, and haplotype frequency distributions of these markers were compared between cases and controls to confirm the associations. The genotype frequency distributions of ADH4 markers were in HWE in EA controls, but were in Hardy-Weinberg disequilibrium (HWD) (ie, deviation from HWE) in EA cases. Among all markers, SNP2 (rs | 042363) at exon 9 or SNP6 (rs | 800759) at the promoter showed the greatest degree of HWD, among patients with either alcohol dependence or drug dependence. Significant differences between EA cases and controls were seen for genotype $\left(10^{-6}<\right.$ global $\left.p<0.044\right)$, but not any allele or haplotype, frequency distributions for all seven ADH4 markers. These findings suggest that $\mathrm{ADH} 4$ genotypes predispose to alcohol dependence and drug dependence in a recessive manner, a predisposition that is population specific. SNP2 or SNP6 was the marker genetically closest to the functional risk loci for both alcohol dependence and drug dependence.

Neuropsychopharmacology (2006) 3 I, I085- 1095. doi: I0.1038/sj.npp. I 300925; published online 12 October 2005
\end{abstract}

Keywords: ADH4 gene; alcohol dependence; drug dependence; Hardy-Weinberg disequilibrium (HWD)

\section{INTRODUCTION}

Ethanol, absorbed through the gastrointestinal tract, enters the liver via the portal vein. After being oxidized in the liver, ethanol metabolites and any unmetabolized ethanol enter the general circulation. Two kinds of enzymes are primarily responsible for the oxidation of alcohol. The first enzyme family is the alcohol dehydrogenases (ADH), which convert alcohol to acetaldehyde (which is toxic). The second enzyme family is the aldehyde dehydrogenases (ALDH), which

\footnotetext{
* Correspondence: Dr J Gelernter, Yale University School of Medicine, VA Psychiatry I I6A2, 950 Campbell Avenue, West Haven, CT 065 I6, USA, Tel: + | 203932 57|| ext. 3590, Fax: + | 2039373897.

E-mail: joel.gelernter@yale.edu

Received 4 April 2005; revised 19 July 2005; accepted 30 August 2005 Online publication: 2 September 2005 at http://www.acnp.org/citations/ Npp090205050219/default.pdf
}

quickly convert acetaldehyde into acetate. Acetate is then oxidized into $\mathrm{CO}_{2}$ and $\mathrm{H}_{2} \mathrm{O}$ via the tricarboxylic acid cycle.

The development of alcohol dependence is related to an individual's ethanol consumption, which is influenced by $\mathrm{ADH}$ activity. Increased $\mathrm{ADH}$ activity can increase the rate of alcohol metabolism, thereby increasing the production of acetaldehyde. If the acetaldehyde cannot be metabolized quickly, the accumulation of acetaldehyde will result in an aversive 'flushing reaction,' characterized by facial flushing, headache, increased skin temperature, dizziness, anxiety, nausea, vomiting, decreased blood pressure with resultant tachycardia and, rarely, severe cardiovascular complications. The aversive effects of acetaldehyde can limit alcohol consumption, and, it is hypothesized, thereby reduce the risk of alcohol dependence (Thomasson et al, 1994; Muramatsu et al, 1995; Hasin et al, 2002).

Active ADH enzyme is composed of two subunits, which can be, but are not necessarily, identical. Humans express 
at least nine possible different $\mathrm{ADH}$ subunits, each with slightly different properties. These isoforms include $\alpha, \beta, \gamma$, $\pi$, $\chi$, ADH6 isoenzyme, $\mu$, and $\sigma$. A unique gene encodes each isoform. A human $A D H$ gene cluster maps to chromosome 4 (4q21-25), extending a total of 364128 base pairs (bp) and including genes encoding five classes, in the following order: IV $(A D H 7), \mathrm{I}-\gamma(A D H 1 C), \mathrm{I}-\beta(A D H 1 B), \mathrm{I}-\alpha$ $(A D H 1 A), \mathrm{V}(A D H 6)$, II $(A D H 4)$, and III $(A D H 5)$.

The human $\pi$ subunit is encoded by the $A D H 4$ gene. In adults, the $\pi$ subunit mainly contributes to liver $\mathrm{ADH}$ activity, catalyzing the oxidation or reduction of a wide range of substrates at moderate and high concentrations, including ethanol, primary long-chain aliphatic alcohols, and aromatic aldehydes (Li et al, 1977; Li and Bosron, 1987; Edenberg et al, 1999). Variation in ADH4 could change the function of the $\pi$ subunit and affect the risk for alcohol dependence by modulating alcohol metabolism. For example, the $-75 \mathrm{~A}$ allele has promoter activity that is more than twice that of the $-75 \mathrm{C}$ allele; this $-75 \mathrm{~A} / \mathrm{C}$ substitution (SNP6: rs1800759) (see Figure 1 for single nucleotide polymorphism (SNP) definitions and locations) at the promoter could significantly affect $\pi$ subunit expression (Edenberg et al, 1999). Considering the important role of the $A D H 4$ gene in alcohol metabolism, it is a functional candidate for alcohol dependence.

Several linkage studies, including the Collaborative Study on the Genetics of Alcoholism (COGA) (Reich et al, 1998) and a study by investigators at the National Institute on Alcohol Abuse and Alcoholism (NIAAA) (Long et al, 1998), have demonstrated that the $A D H$ gene cluster is coincident with a possible linkage signal for alcohol dependence. Reich et al (1998) performed a genome-wide screen by examining 291 markers in 987 individuals from 105 affected families and found suggestive evidence of a protective locus for alcohol dependence on chromosome 4 near the $A D H$ gene cluster. Zinn-Justin and Abel (1999) performed an autosome-wide scan in the COGA data using the method of weighted pairwise correlation incorporating identityby-descent (WPC-IBD), and located a risk locus for alcohol dependence at a $50 \mathrm{cM}$ region spanning from $G A B R B 1$ to D4S1651, a region harboring the $A D H$ genes. Williams et al (1999) applied a variance-component method to analyze COGA data and located a risk locus for alcohol dependence at a chromosome 4 region near the $A D H$ gene cluster. Saccone et al (2000) carried out HasemanElston-based regression analyses on the COGA data set and mapped susceptibility genes for a quantitative alcoholism-related phenotype, that is, 'maximum number of drinks consumed in a 24-h period,' which is correlated with the alcohol dependence diagnosis, to the region of the $A D H$ gene cluster. In the study from NIAAA, Long et al (1998) performed a whole-autosomal genome scan with 517 microsatellite markers in several extended pedigrees and found that three loci in the $A D H$ gene cluster showed evidence for linkage with alcohol dependence. In summary, the $A D H$ gene cluster is in a region that has been positionally implicated in alcohol dependence. Since the $A D H 4$ gene maps within this $A D H$ gene cluster, ADH4 gene is also a positional candidate for alcohol dependence.

There are also case-control association data implicating the same region of chromosome 4 (but different specific loci) by showing associations of $A D H$ genes to alcohol dependence (eg, Thomasson et al, 1994; Hasin et al, 2002; Konishi et al, 2003). Foroud et al (2003) analyzed 58 markers at the $A D H$ gene cluster in COGA alcoholic families using the pedigree disequilibrium test and primarily identified eight variants (published abstract only; specific marker names were unavailable to us) in the $A D H 4$ gene associated with the risk for alcohol dependence in European-Americans (EAs).

The present study aimed to extend these findings by examining the relationship between $A D H 4$ gene variants and alcohol dependence in several populations, including EAs and African-Americans (AAs). We focused on the $A D H 4$ gene in this study because of the functional importance of its protein product, remarkable for its activity at high ethanol concentrations that might be of particular relevance in the context of alcohol dependence risk (Li et al, 1977); because of the preliminary COGA results (Foroud et al, 2003); and because our preliminary case-control survey of variants at many $\mathrm{ADH}$ loci immediately identified this as a gene of interest (Luo et al, unpublished data).

Drug dependence, which mainly includes cocaine dependence and opioid dependence in our sample, is one of the most common phenotypes comorbid with alcohol dependence (Gossop, 2004). Many studies have demonstrated that drug dependence has a number of features in common with alcohol dependence, including symptomatology (Glatt, 1967; Heil et al, 2001), neuropsychological impairments (Robinson et al, 1999), pathogenetic mechanisms (Anokhina et al, 1990; O’Neill et al, 2001), and response to specific treatments, especially the effects of disulfiram, an ALDH blocker, which though approved for the treatment of alcohol dependence, has also been shown to be efficacious in the treatment of cocaine dependence (Carroll et al, 1993, 1998, 2000, 2004; George et al, 2000; Petrakis et al, 2000). Drug dependence may share susceptibility genes with alcohol dependence (reviewed by Kreek et al, 2004), for example, OPRM1 gene variation moderated susceptibility to alcohol dependence and/or drug dependence (Hoehe et al, 2000; Szeto et al, 2001; Schinka et al, 2002; Luo et al, 2003). To answer the question of whether $A D H 4$ variants influence risk for alcohol dependence, drug dependence, or both of these traits, we examined the association of $A D H 4$ gene with drug dependence diagnoses in this study.

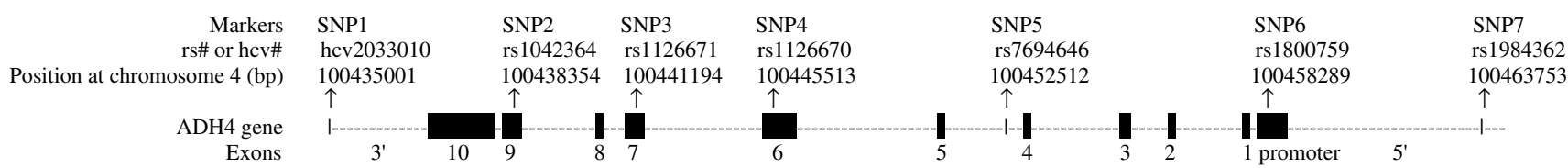

Figure I Map of markers at the $\mathrm{ADH} 4$ gene. 


\section{MATERIALS AND METHODS}

\section{Subjects}

A total of 926 unrelated subjects (557 male and 369 female subjects), including $400 \mathrm{EA}$ cases, $317 \mathrm{EA}$ healthy controls, $161 \mathrm{AA}$ cases, and $48 \mathrm{AA}$ controls, were included in this study. The cases met lifetime DSM-IV criteria (American Psychiatric Association, 1994) for alcohol dependence, drug (cocaine or opioid) dependence, or a combination of these disorders. Among the cases, 436 subjects had a diagnosis of alcohol dependence (334 EAs, 102 AAs) and 356 had a diagnosis of drug dependence (207 EAs, 149 AAs), including 318 with cocaine dependence (178 EAs, 140 AAs) and 152 with opioid dependence (95 EAs, 57 AAs). The control subjects were screened to exclude major Axis I mental disorders, including alcohol or drug abuse or dependence, psychotic disorders (including schizophrenia or schizophrenia-like disorders), mood disorders, and anxiety disorders. Male subjects constituted $73.2 \%$ of the cases and $40.0 \%$ of the controls. The average ages were $39.4 \pm 9.2$ years for cases and $28.1 \pm 9.1$ years for controls.

The subjects were recruited at the University of Connecticut Health Center or the VA Connecticut Healthcare System-West Haven Campus. All subjects gave informed consent before participating in the study, which was approved by the Institutional Review Board at the respective institutions.

\section{Genotyping}

Marker inclusion (see Figure 1). Seven known SNPs (all of those available from the public Applied Biosystems (Foster City, CA) website (http://www.appliedbiosystems.com/) at the time of genotyping; designated by hcv\# or rs\#) were included in this study (see Figure 1). These SNPs were selected based on the following findings or hypotheses: (1) the promoter SNP (SNP6: rs1800759) is known to be a functional polymorphism, altering promoter activity (Edenberg et al, 1999); (2) SNP2 (rs1042363) at exon 9 and SNP3 (rs1126671) at exon 7, which result in the aminoacid substitutions $\mathrm{Gly} \rightarrow \mathrm{Arg}$ and $\mathrm{Val} \rightarrow$ Ile, respectively, might also affect the function of the $\pi$ subunit; and (3) some 'silent' SNPs such as SNP1 (hcv2033010) at the $3^{\text {' end, }}$ SNP4 at exon 6, SNP5 (rs7694646) at intron 4, and SNP7 (rs1984362) at the $5^{\prime}$ end might influence $\pi$ subunit expression as well, either directly (eg, through effects on regulation or mRNA stability) or indirectly via linkage disequilibrium (LD) with other functional variants such as SNP2, SNP3, or SNP6.

The allele frequencies and PCR conditions for these markers have been validated by ABI. The heterozygosity of each of these markers in the ABI database is $>0.375$ in an EA population, which corresponds to a minor allele frequency $>0.25$. These markers, spanning the full length of $A D H 4$ gene, cover a range of $28752 \mathrm{bp}$, with an average spacing of $4792 \mathrm{bp}$.

Genotyping. Genomic DNA was extracted from peripheral blood by standard methods. The seven ADH4 SNPs were genotyped with a fluorogenic $5^{\prime}$ nuclease assay method, that is, the TaqMan technique (Shi et al, 1999), using the ABI PRISM $^{\circledR} 7900$ Sequence Detection System (ABI, Foster City, CA, USA). PCR was performed in a final volume of $3 \mu$, including $1.5 \mu \mathrm{l} 2 \times$ TaqMan Universal PCR Master Mix (ABI), $0.075 \mu \mathrm{l} 20 \times$ Assays-on-Demand or $0.0375 \mu \mathrm{l} 40 \times$ Assays-by-Design (ABI), $0.03 \mu \mathrm{l} 100 \times \mathrm{BSA}$ (New England Biolabs Inc., Beverly, MA), and 1 ng DNA. All genotyping was performed in duplicate and compared to ensure validity of the data. Mismatched genotypes, which constituted $<0.5 \%$ of the total number of duplicate genotypes performed, were discarded.

\section{Statistical Analysis}

Linkage disequilibrium analysis. Different populations have different generations of ancestries and different degrees of admixture, which may result in different LD decays from the initial generations to the current generations. Different LD decay is one factor that results in different LD block sizes between populations. Thus, in the present study, the pairwise LD between any two $A D H 4$ markers was analyzed separately for EAs and AAs. The $D^{\prime}$ value for each LD pair was calculated and visualized through the program Haploview (Barrett et al, 2005) (see Figure $2 \mathrm{a}$ and $\mathrm{b}$ ).

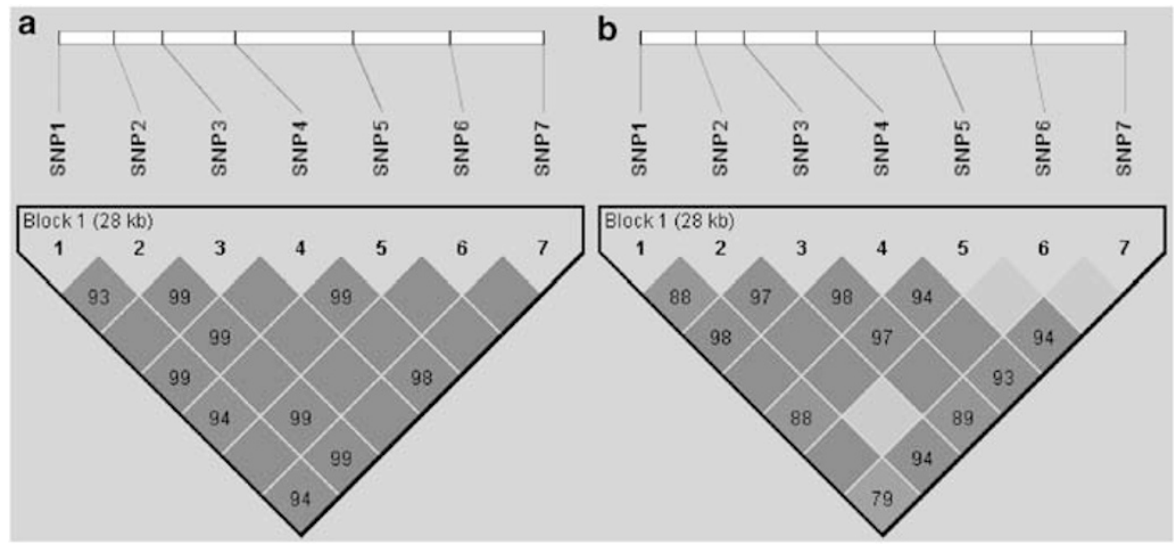

Figure 2 LDs among seven ADH4 markers in (a) unrelated EA subjects and (b) unrelated AA subjects. $D^{\prime}=1.00$ in the blank red squares; the numbers inside the darker squares are $D^{\prime} \times 100 \%$; the lighter squares represent low $r^{2}$ values. 
Hardy-Weinberg equilibrium test. Deviation from HardyWeinberg equilibrium (HWE) expectations, that is, HardyWeinberg disequilibrium (HWD), can indicate a valid gene-phenotype association (Feder et al, 1996; Nielsen et al, 1998; Jiang et al, 2001; Hoh et al, 2001; Lee, 2003; Hao et al, 2004). The HWD test as an association method in cases only has been reported to be more powerful than a case-control study, especially for a recessive model (Nielsen et al, 1998). HWE was tested within populations, and separately for cases and controls. The test in a mixed population was conducted as an exploratory evaluation of population stratification effects. HWE tests in the groups with mixed affection status (ie, those resulting from combining cases and controls) were used to determine whether the markers were in HWE within each population. HWE for each marker was tested using goodness-of-fit implemented in the program PowerMarker (Liu and Muse, 2004). Genotype frequency distributions of the seven markers within each phenotype group were also considered together and tested by an omnibus HWD test via the program 'min_p_test' (see below).

In addition to the use of the HWD test to evaluate associations between genes and diseases, the HWD test must be applied to determine what analysis methods (software programs) are valid for haplotype reconstruction (see below), because some methods are valid only in the presence of HWE.

Two HWD measures (ie, $F$ and $J$ ) can be used to fine-map the disease risk locus. These measures are calculated using the following equations: $F=\Delta / H_{\mathrm{e}}$ (Feder et al, 1996) and $J=\Delta / 2 H_{\mathrm{e}}^{\prime}$ (Jiang et al, 2001), where $\Delta=H_{\mathrm{e}}-H_{\mathrm{o}}, H_{\mathrm{e}}$ and $H_{\mathrm{o}}$ are the expected and the observed frequencies, respectively, of the heterozygotes in cases (assuming HWE), and $H_{\mathrm{e}}^{\prime}$ is the expected frequency of the non-disease-associated homozygotes in cases. $F$ and $J$ are decreasing functions of $\theta$, where $\theta$ is the recombination rate between the marker and the disease susceptibility locus. Higher values of $F$ and $J$ reflect greater LD between marker and disease locus. The marker locus with either the highest $F$ or $J$ value was taken as the marker closest to the disease susceptibility locus. The value of $F$ depends not only on the genetic distance between the marker locus of interest and the disease susceptibility locus, but also on the allele frequencies at the marker locus. $J$ does not hinge on the allele frequencies at the marker locus.

Case-control comparisons for allele and genotype frequencies. The allele and genotype frequencies of the $A D H 4$ markers among EAs and AAs are shown in Tables 1 and 2. Associations between the alleles, genotypes, and phenotypes were tested by comparing the allele and genotype frequency distributions between cases and controls with the exact tests in the program PowerMarker.

To avoid type I error due to multiple comparisons without information loss due to the overly conservative Bonferroni correction, the genotypes of these seven markers were considered together in an omnibus test using the program 'min_p_test' (Hao et al, 2004; personal communication), to obtain adjusted global $p$-values and potentially to identify real susceptibility SNPs among these markers. 'min_p_test' provides three $p$-values based on three different statistics including the Pearson $\chi^{2}$ test statistic (PE) for genotypewise analysis, the Cochran-Armitage $\chi^{2}$ trend test statistic (CA) for allelewise analysis, and the HWD statistic for HWD test.

\section{Haplotype Frequency Analysis}

Multilocus haplotypes usually are more informative than any single marker. However, the $A D H 4$ markers are in HWD in cases, violating the assumption of the expectationmaximization (EM) algorithm upon which many haplotypereconstruction programs are based. The Bayesian approach and the Partition Ligation algorithm may still be applied to reconstruct haplotypes even when HWE is violated (Niu et al, 2002). The program PHASE 2.0.2, based on a combination of the Bayesian statistical method and the socalled 'divide-and-conquer' (ie, Partition Ligation) strategy, which is claimed to be more accurate in reconstructing haplotypes than the EM algorithm, was used for this purpose (Stephens et al, 2001; Stephens and Donnelly, 2003). PHASE not only provides the overall haplotype frequency distribution in the whole sample, but also directly compares the haplotype frequency distributions between cases and controls.

The haplotype frequency distribution depends on the LD among markers, which varies by population. In order to infer the haplotypes more accurately, haplotypes were reconstructed separately for EAs and AAs.

\section{RESULTS}

(1) Pairwise analysis in different populations showed that the ADH4 markers were in strong LD. In EAs, the seven $A D H 4$ markers were in strong LD $\left(D^{\prime}>0.9\right)$ (see Figure 2a). In AAs, six $A D H 4$ markers were in strong LD $\left(D^{\prime}>0.9\right)$, with SNP1 in weaker LD with the others $\left(D^{\prime}>0.79\right)$ (Figure $2 b$ ). These seven markers can be taken as representing a single haplotype block (Gabriel et al, 2002). There were no significant differences in LD among the $A D H 4$ markers between cases and controls (within each population).

(2) The genotype frequency distributions of the seven ADH4 markers were in HWD in the EA patients with alcohol dependence or cocaine dependence, but were in HWE in the EA healthy controls (see Table 3).

In EAs, the genotype frequency distributions of all seven ADH4 markers were in HWD in patients with alcohol dependence or cocaine dependence, but were in HWE in patients with opioid dependence and in controls. After Bonferroni correction, where $\alpha$ was set at $0.007(=0.05 / 7 ; 7$ is the number of markers), five markers (SNP2-6) and seven markers remained in HWD in patients with alcohol dependence and cocaine dependence, respectively. SNP2 was in HWD in subjects with drug dependence without comorbid alcohol dependence, even after Bonferroni correction. As mentioned above, the Bonferroni correction is very conservative, as the markers were in LD and their genotypes were correlated. The omnibus HWD test, which obviates the Bonferroni correction, showed that the genotype frequency distributions of $A D H 4$ markers were in HWD in all affected groups of EAs (see global $p$-values for HWD tests in Table 3). 
Table I Genotype and Allele Frequencies of Seven ADH4 Markers in EAs

\begin{tabular}{|c|c|c|c|c|c|c|c|c|c|c|c|c|}
\hline & \multicolumn{2}{|c|}{$A D(n=334)$} & \multicolumn{2}{|c|}{$\operatorname{Coc}(n=178)$} & \multicolumn{2}{|c|}{ Op $(n=95)$} & \multicolumn{2}{|c|}{$\mathrm{DD}(n=207)$} & \multicolumn{2}{|c|}{ DD-only $(n=66)$} & \multicolumn{2}{|c|}{ Con $(n=3 \mid 7)$} \\
\hline & $\mathbf{N}$ & $f_{o}$ & $N$ & $f_{o}$ & $N$ & $f_{o}$ & $\mathbf{N}$ & $f_{o}$ & $N$ & $f_{o}$ & $N$ & $f_{o}$ \\
\hline \multicolumn{13}{|l|}{ SNPI } \\
\hline $\mathrm{T} / \mathrm{T}$ & 38 & 0.119 & 25 & 0.144 & 10 & 0.108 & 26 & 0.129 & 10 & 0.152 & 24 & 0.078 \\
\hline $\mathrm{T} / \mathrm{C}$ & 116 & 0.363 & 55 & 0.316 & 30 & 0.323 & 65 & 0.323 & 24 & 0.364 & 140 & 0.458 \\
\hline$C$ & 448 & 0.700 & 243 & 0.698 & 136 & 0.731 & 285 & 0.709 & 88 & 0.667 & 424 & 0.693 \\
\hline \multicolumn{13}{|l|}{ SNP2 } \\
\hline $\mathrm{T} / \mathrm{T}$ & 34 & 0.107 & 26 & 0.149 & 9 & 0.098 & 27 & 0.135 & 12 & 0.185 & 18 & 0.060 \\
\hline $\mathrm{T} / \mathrm{C}$ & 107 & 0.338 & 46 & $0.264^{\mathrm{a}}$ & 27 & $0.293^{\mathrm{a}}$ & 54 & $0.270^{\mathrm{a}}$ & 20 & $0.308^{a}$ & $|3|$ & 0.440 \\
\hline \multicolumn{13}{|l|}{ SNP3 } \\
\hline $\mathrm{A} / \mathrm{A}$ & 44 & 0.135 & 26 & 0.148 & 10 & 0.105 & 27 & 0.132 & 10 & 0.154 & 24 & 0.078 \\
\hline $\mathrm{A} / \mathrm{G}$ & 119 & 0.365 & 56 & 0.318 & 32 & 0.337 & 67 & 0.328 & 24 & 0.369 & 147 & 0.476 \\
\hline $\mathrm{G} / \mathrm{G}$ & 163 & 0.500 & 94 & 0.534 & 53 & 0.558 & 110 & 0.539 & 31 & 0.477 & 138 & 0.447 \\
\hline A & 207 & 0.317 & 108 & 0.307 & 52 & 0.274 & 121 & 0.297 & 44 & 0.338 & 195 & 0.316 \\
\hline G & 445 & 0.683 & 244 & 0.693 & 138 & 0.726 & 287 & 0.703 & 86 & 0.662 & 423 & 0.684 \\
\hline \multicolumn{13}{|l|}{ SNP4 } \\
\hline C/C & 43 & 0.134 & 25 & 0.146 & 10 & 0.108 & 26 & 0.131 & 10 & 0.159 & 24 & 0.077 \\
\hline $\mathrm{A} / \mathrm{C}$ & 114 & 0.356 & 53 & 0.310 & 30 & 0.323 & 63 & 0.318 & 23 & 0.365 & 145 & 0.468 \\
\hline A & 184 & 0.284 & 96 & 0.276 & 47 & 0.250 & 108 & 0.267 & 42 & 0.323 & 175 & 0.283 \\
\hline $\mathrm{T}$ & 464 & 0.716 & 252 & 0.724 & $|4|$ & 0.750 & 296 & 0.733 & 88 & 0.677 & 443 & 0.717 \\
\hline \multicolumn{13}{|l|}{ SNP6 } \\
\hline $\mathrm{A} / \mathrm{A}$ & 67 & 0.209 & 39 & 0.223 & 15 & 0.163 & 40 & 0.199 & 16 & 0.242 & 44 & 0.145 \\
\hline $\mathrm{A} / \mathrm{C}$ & 122 & $0.381^{a, b}$ & 60 & $0.343^{b}$ & 35 & $0.380^{b}$ & 74 & $0.368^{b}$ & 25 & $0.379^{b}$ & 154 & 0.507 \\
\hline $\mathrm{C} / \mathrm{C}$ & 131 & 0.409 & 76 & 0.434 & 42 & 0.457 & 87 & 0.433 & 25 & 0.379 & 106 & 0.349 \\
\hline A & 256 & 0.400 & 138 & 0.394 & 65 & 0.353 & 154 & 0.383 & 57 & 0.432 & 242 & 0.398 \\
\hline C & 384 & 0.600 & 212 & 0.606 & 119 & 0.647 & 248 & 0.617 & 75 & 0.568 & 366 & 0.602 \\
\hline \multicolumn{13}{|l|}{ SNP7 } \\
\hline $\mathrm{A} / \mathrm{A}$ & 35 & 0.108 & 22 & 0.127 & 9 & 0.096 & 23 & 0.114 & 10 & 0.156 & 20 & 0.065 \\
\hline$A / G$ & 110 & 0.340 & 50 & 0.289 & 29 & 0.309 & 60 & 0.299 & 22 & 0.344 & 134 & 0.434 \\
\hline $\mathrm{G} / \mathrm{G}$ & 179 & 0.552 & $10 \mid$ & 0.584 & 56 & 0.596 & 118 & 0.587 & 32 & 0.500 & 155 & 0.502 \\
\hline A & 180 & 0.278 & 94 & 0.272 & 47 & 0.250 & 106 & 0.264 & 42 & 0.328 & 174 & 0.282 \\
\hline G & 468 & 0.722 & 252 & 0.728 & $|4|$ & 0.750 & 296 & 0.736 & 86 & 0.672 & 444 & 0.718 \\
\hline
\end{tabular}

AD, alcohol dependence; Coc, cocaine dependence; Op, opioid dependence; DD, drug dependence (ie, cocaine dependence and/or opioid dependence); DD-only, drug dependence without comorbid alcohol dependence; Con, controls; $n$, sample size; $N$, individual numbers (for genotype) or chromosome numbers (for alleles); fo, observed genotype or allele frequency.

${ }^{a}$ The marker with highest $F$ value among all markers. $F=\left(H_{e}-H_{0}\right) / H_{e}$, where $H_{e}$ and $H_{0}$ are the expected and the observed frequencies of heterozygote of a marker.

${ }^{b}$ The marker with highest $J$ value among all markers. $J=\left(H_{\mathrm{e}}-H_{\mathrm{o}}\right) / 2 \mathrm{H}_{\mathrm{e}}^{\prime}$, where $H_{\mathrm{e}}^{\prime}$ is the expected frequency of the non-disease-associated homozygote of a marker. 
Table 2 Genotype and Allele Frequencies of Seven ADH4 Markers in AAs and HWD Tests in AAs, EAs and EAs+AAs

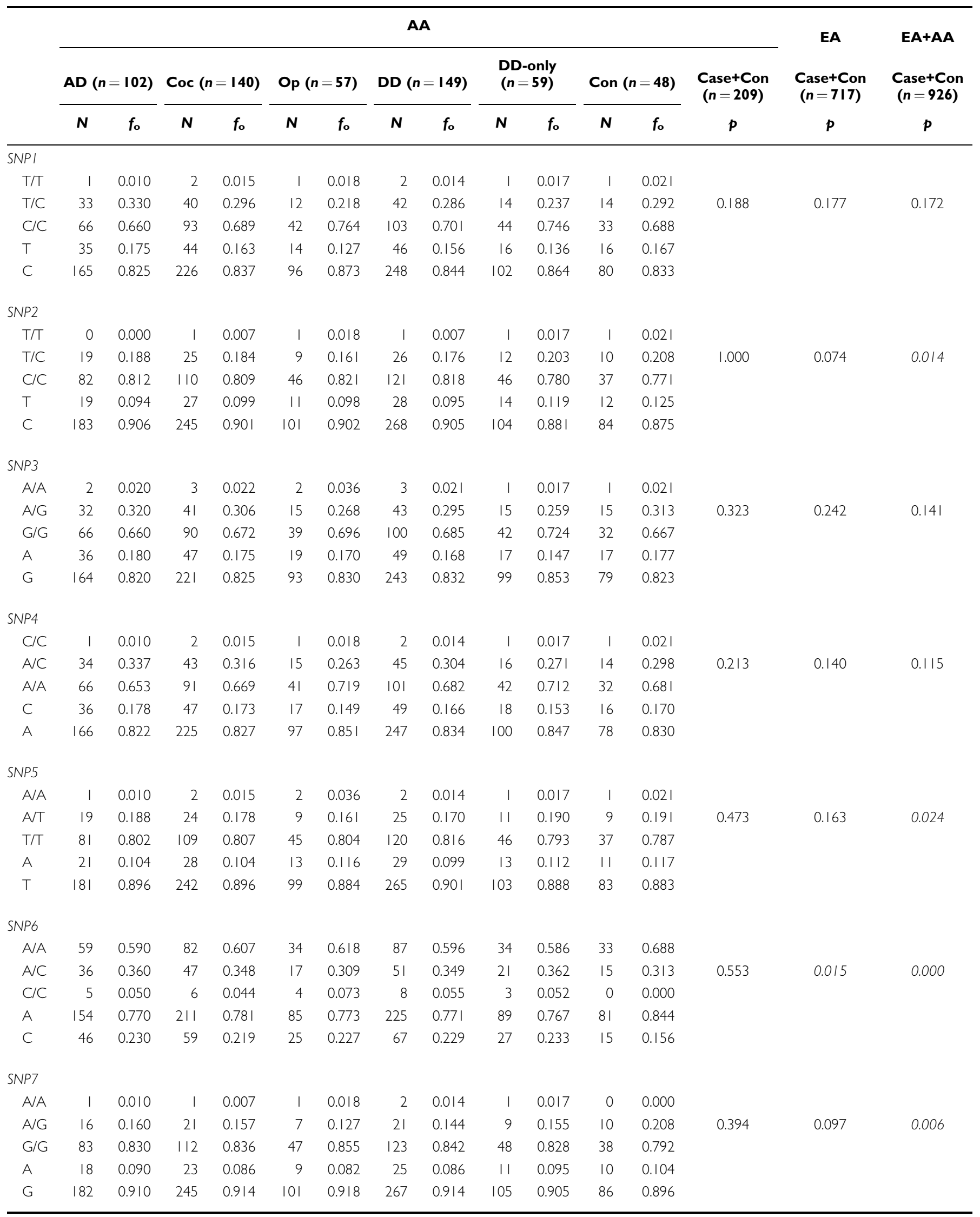

AA, African-Americans; EA, European-Americans; AD, Coc, Op, DD; DD-only, Con, $n, N$, fo, see Table I footnote; Case, all patients; $p$, $p$-value for HWE test (goodness-of-fit). Italic numbers denote $p<0.05$. 


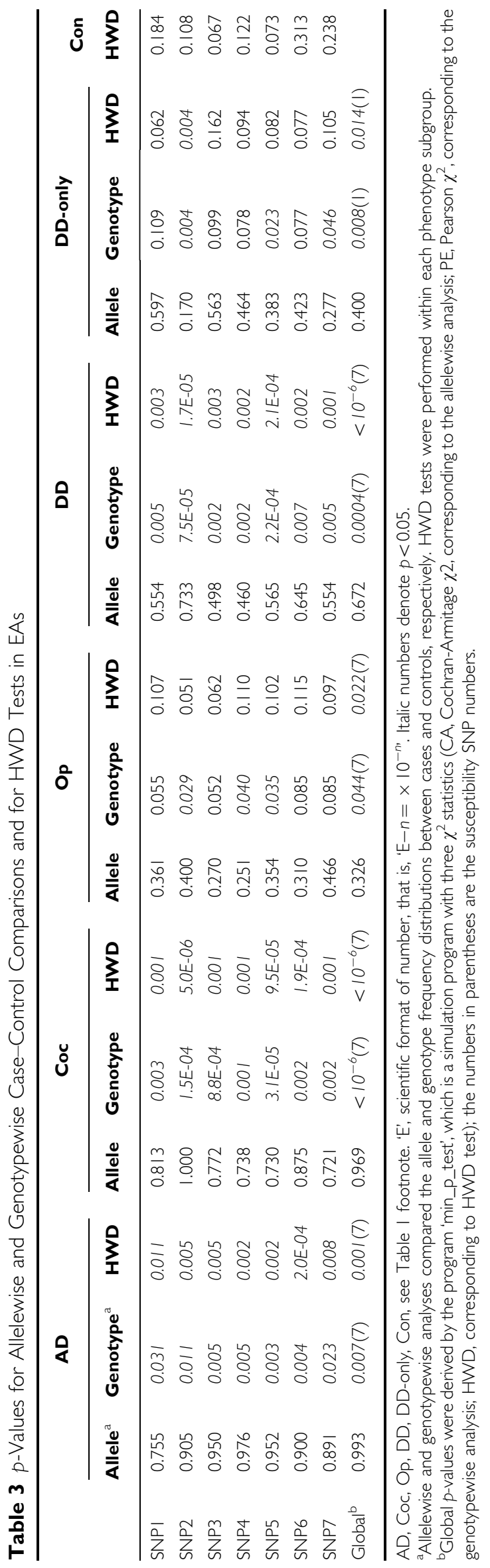

Among these markers, SNP6 and SNP2 were in most significant HWD in cases with alcohol dependence and cocaine dependence, respectively (see Table 3). A goodnessof-fit test showed that these deviations from HWE were attributable to the excess of the observed homozygote frequency $\left(f_{\mathrm{o}}\right)$ (see Table 1 ) over the expected homozygote frequency $\left(f_{\mathrm{e}}\right)$ (data not shown).

According to the values of $F$, SNP6 and SNP2 were the markers closest to the risk loci for alcohol dependence and drug dependence (including cocaine and opioid dependence), respectively. But after correcting for the effects of allele frequencies of markers on HWD, that is, as reflected in the values of $J$, SNP6 is the marker closest to the risk loci for both alcohol dependence and drug dependence ( $J$ values not shown).

In AAs, all of the markers were in HWE in patients and controls. Although SNP5 and SNP7 were nominally in HWD in patients with opioid dependence $(p=0.020$ and 0.029 , respectively), the effects were not significant after Bonferroni correction (see Table 2, $p$-values not listed).

Within EAs and AAs (mixing cases and controls; see second-to-last and third-to-last columns in Table 2), most ADH4 markers were in HWE, except SNP6 in EAs $(p=0.015)$. When mixing EAs and AAs (see the last column in Table 2), over half of these markers were in HWD, which was expected given that the population was not stratified.

(3) The genotypes of $A D H 4$ markers were associated with alcohol dependence and drug dependence in EAs ( $p$-values listed in Table 3).

The allele and genotype frequencies of the seven $A D H 4$ markers are listed in Tables 1 and 2. In EAs, there were nominally significant differences in genotype, but not in any allele, frequency distributions between controls and subjects with alcohol dependence, cocaine dependence, opioid dependence, or any drug dependence without comorbid alcohol dependence (see Table 3). All of these associations resulted from the higher frequency of homozygotes of each marker in cases than in controls (see Table 1). Before Bonferroni correction, the genotypes of all seven SNPs were associated with alcohol dependence and cocaine dependence $(p<0.05)$; three SNPs, that is, SNP2, SNP4, and SNP5, were associated with opioid dependence; and three SNPs, that is, SNP2, SNP5, and SNP7, were associated with drug dependence without comorbid alcohol dependence. After Bonferroni correction $(\alpha=0.05 / 7=0.007)$, four SNPs (ie, SNP3-6) were associated with alcohol dependence, all seven SNPs were associated with cocaine dependence, and one SNP (ie, SNP2) was associated with drug dependence without comorbid alcohol dependence.

The omnibus tests using the 'min_p_test' program showed compelling evidence that (1) all seven SNPs were susceptibility markers for both alcohol dependence $\left(P_{\mathrm{PE}}=0.007\right)$ and drug dependence $\left(P_{\mathrm{PE}}=0.0004\right)$ (highly significant in cocaine dependence $\left(P_{\mathrm{PE}}<10^{-6}\right)$ and modestly significant in opioid dependence $\left.\left(P_{\mathrm{PE}}=0.044\right)\right)$, and (2) one of the SNPs (SNP2) was a susceptibility marker for drug dependence without comorbid alcohol dependence $\left(P_{\mathrm{PE}}=0.008\right)$, an association identified despite the small sample size available for this subgroup.

In AAs, no significant differences were found in either allele or genotype frequencies between cases and controls. 
(4) The program PHASE was used to compute estimated haplotype frequency distributions and directly compare these distributions between cases and controls. There were no significant differences in the seven-variant haplotype frequency distributions between cases and controls, in either EAs or AAs (data not shown, all $p>0.05$ ).

\section{DISCUSSION}

The present study provides strong evidence supporting the hypothesis that $A D H 4$ is a susceptibility gene for alcohol dependence, and presents the first evidence that it is also a susceptibility gene for drug dependence. These findings were obtained using the HWD test in a case-only design and were confirmed using a case-control design.

In this study, the average age for controls was 28.1 years, more than 10 years younger than that (39.4 years) for cases. Many healthy controls have not completely passed the risk age for manifesting alcohol dependence or drug dependence. The healthy controls have a probability $(\approx$ prevalence of disease) to develop disease in their lifetime, and this probability increases when the prevalence is moderate (eg, alcohol dependence or major depression), so that the case-control association design becomes less powerful than a case-only study. That is, some associations that can be discovered by the use of a case-only study might not be detected using a case-control design. This is due to the fact that there are likely to be some control subjects who, although presently unaffected, have the genetic liability for affection and may be affected in the future. Therefore, under these circumstances, the case-only study design is more sensitive for the detection of allelic association between susceptibility alleles and diseases. The HWD test is an association method for case-only study, which has been demonstrated to be more powerful than case-control study in some circumstances. This seems to be particularly true for recessive diseases (Nielsen et al, 1998). Because, to our knowledge, this method has not been previously employed in psychiatric genetics (except in one previous study, Luo et al, 2005), it was necessary to confirm the results obtained with this method using a conventional case-control association study as a positive control.

A disease has the potential to exert strong selection on the genome, which can result in HWD for some specific loci. This deviation from HWE could be very significant if it occurs right at the disease risk locus (Nielsen et al, 1998; Wittke-Thompson et al, 2005). The markers in LD with the risk locus may be in HWD as well; alternatively, HWD may indicate that the markers in HWD are in close proximity to the disease risk locus. The detection of HWD has recently been shown to be useful as a method for detecting genephenotype association (Feder et al, 1996; Nielsen et al, 1998; Jiang et al, 2001; Hoh et al, 2001; Lee, 2003; Hao et al, 2004; Wittke-Thompson et al, 2005; Luo et al, 2005). The present study provides an additional illustration of this approach. We found that the ADH4 markers were in HWE in EA healthy controls, but were in HWD in EA cases with alcohol dependence or drug (ie, cocaine or opioid) dependence, which strongly suggests that the HWD reflects a gene-phenotype association. When considering all the markers together in an omnibus test (using the program 'min_p_test') to avoid multiple comparisons, we obtained global $p$-values that ranged from $10^{-6}$ to 0.022 for HWD tests in all phenotype groups (see $P_{\mathrm{HWD}}$ in Table 3 ), which is a strong indication that $A D H 4$ is in LD with the putative disease risk locus (or may be a risk locus itself). In other words, there are strong associations between diseases (including alcohol dependence and drug dependence) and ADH4 in EAs.

To confirm the findings we obtained using HWD tests, we also compared cases with controls on the allele, genotype, and haplotype frequency distributions within EA and AA populations. We found that (1) the allelewise analysis (using the program PowerMarker) did not detect any significant difference in the allele frequency distribution for any marker between cases and controls within EAs or AAs. Considering all of the markers together in an omnibus test to avoid multiple tests, we obtained global $p$-values $>0.05$ for all allelewise analyses (see $P_{\mathrm{CA}}$ in Table 3). These negative results may be attributable to HWD, because under HWD, the marker alleles are not independent of one another, which leads to the case that the effects of diseasepredisposing alleles may be 'masked' by other non-diseasepredisposing alleles (Cordell, 2002). This reduces the power for the allelewise analysis (Sasieni, 1997; Nielsen et al, 1998; Luo et al, 2005) and may even invalidate it. (2) A multilocus haplotype is actually the subset of every single-locus allele; both allele and haplotype reflect the features of chromosomes in the population. Similar to the allelewise analysis, the haplotypewise analysis (using the program PHASE) in this study showed significant differences in the haplotype frequency distributions between cases and controls neither in EAs nor in AAs. Although these negative results (directly derived from the program PHASE) might be partly attributable to the low power of PHASE for case-control comparison, we speculate that they more likely result from HWD, because under HWD, as with the allelewise analysis, the haplotypes are not independent of one another. Consequently, disease-predisposing haplotypes may be 'masked' by other non-disease-predisposing haplotypes, which thus reduces the power for, or even invalidates, the haplotypewise analysis. We have also demonstrated that the haplotypewise analysis is potentially less powerful than the diplotypewise analysis (Luo et al, 2005). A diplotype (ie, a haplotype pair) is the subset of every single-locus genotype; both genotype and diplotype represent the types of chromosome pairs in each individual. Diplotype is predicted by PHASE too, but diplotypewise analysis is exempt from the HWE requirement and thus preserves power. (3) Genotypewise analysis is valid regardless of the presence of HWE and thus should preserve statistical power even in the presence of strong HWD. Very interestingly, as predicted, the genotypewise analysis showed that there were significant differences in genotype frequency distributions for all seven ADH4 markers between cases and controls in EAs (see Table 3). After Bonferroni correction, the genotypes of four $A D H 4$ markers were significantly associated with alcohol dependence; seven markers were associated with drug dependence (mainly cocaine dependence); and one marker was associated with drug dependence without comorbid alcohol dependence. Bonferroni correction treats every association test as an independent test, but since the seven $A D H 4$ markers are in strong $\mathrm{LD}$, this 
approach is clearly too conservative for setting the significance level, which could result in information loss. To avoid multiple comparisons and the concomitant inflation of type I error, we considered all of the markers together in an omnibus test using the program 'min_p_test'; this approach identified seven susceptibility markers for alcohol dependence (global $P_{\mathrm{PE}}=0.007$ ) and drug dependence (cocaine dependence, global $P_{\mathrm{PE}}<10^{-6}$; opioid dependence, global $P_{\mathrm{PE}}=0.044$ ) and one susceptibility marker for drug dependence without alcohol dependence (global $P_{\mathrm{PE}}=0.008$ ). These associations are population specific, that is, they were seen only in EAs, not in AAs. (We did not exclude the possibility that other unknown ADH4 markers might be associated with alcohol dependence or drug dependence in AAs, and did not exclude the possibility that negative results in AAs are attributable to small sample size for that population.) Therefore, in the present study, the allele and haplotype analyses were considered exploratory, and we emphasized the genotypewise analysis, whence we drew conclusions.

Comparing the genotypewise analyses and the HWD tests, we find that (1) the findings from the genotypewise analyses basically coincide with those obtained from the HWD tests. That is, the findings in the case-only study are confirmed by the case-control association study, which is post hoc evidence that the HWD test is a valid association method, and which is also post hoc evidence that makes other explanations (except for this indication of association) for HWD highly unlikely. (2) Most associations from the HWD tests are more significant than those from the case-control comparisons (with lower $p$-values - compare the $p$-values between two tests or compare $P_{\mathrm{HWD}}$ with $P_{\mathrm{PE}}$ in Table 3). In other words, the HWD method can be more powerful than the case-control association method, supporting the conclusion by Nielsen et al (1998).

The fact that the ADH4 markers were in HWE in healthy controls but in HWD in cases not only indicates a genephenotype association, but is also consistent with a recessive mode of inheritance of these markers (Feder et al, 1996; Nielsen et al, 1998). The fact that the associations were seen between the genotypes and the phenotypes, but not between the alleles and the phenotypes, also suggests a recessive mode of inheritance of these susceptibility markers, because when a trait-associated marker acts via a recessive mode of inheritance and is under HWD, the effect of the recessive allele (ie, the diseaserisk allele) can be 'masked' by the dominant allele (ie, the non-disease-risk allele).

Both the HWD test and the case-control design as association methods are potentially vulnerable to population stratification, including admixture effects, which could result in spurious associations. For example, when EAs and AAs are mixed, over half of the markers are observed to be in HWD (see the last column in Table 2), which results from population stratification. Thus, we guarded against this confound by testing for HWD and making comparisons only within each population. Additionally, in a second study (Luo et al, in press), we used the structured association method to stratify our sample, and obtained results consistent with those reported here. Similarly, in the follow-up study (Luo et al, in press), the associations from the present case-only and case-control studies were confirmed using the transmission disequilibrium test (TDT) in an independent sample of small nuclear families.

An outlier marker with extreme HWD usually results from genotyping error, so HWD has also been used to detect such errors (Hoh et al, 2001). However, the genotyping technique applied in this study has a low measured error rate $(<0.5 \%)$ and all the genotyping was performed in duplicate. Furthermore, none of the seven markers is an apparent outlier according to the HWD statistics, so HWD resulting from genotyping error is not a likely explanation for the observed results.

HWD in cases can also be useful for fine-mapping a disease locus. According to the $F$ values (Feder et al, 1996), which reflect the extent of HWD, SNP6 and SNP2 were the markers closest to the risk loci for alcohol dependence and drug dependence (including cocaine and opioid dependence), respectively. After correcting for the influence of marker allele frequency on HWD, that is, according to the $J$ values (Jiang et al, 2001), which also reflect the extent of HWD and are equivalent to $P_{\text {excess }}$ in a case-control design (Devlin and Risch, 1995), SNP6 is the marker closest to the risk loci for both alcohol dependence and drug dependence.

Association between the $A D H 4$ gene and alcohol dependence accords with the functional role of the $\pi$ subunit in the development of alcohol dependence. However, we noted that drug dependence was also associated with $A D H 4$ gene polymorphisms. Interestingly, both in the HWD test and in the case-control comparison, the association of $A D H 4$ gene with drug dependence reached levels of statistical significance that were at least as great as those for alcohol dependence. This suggests two major possibilities: (1) the susceptibility to drug dependence that is attributable to $A D H 4$ is shared with alcohol dependence susceptibility (ie, it is independently a risk factor for each of the two phenotypes); or (2) the associations between the $A D H 4$ gene and drug dependence are driven by the actual associations between $A D H 4$ and alcohol dependence, because many patients with drug dependence have comorbid alcohol dependence. In addressing this question, we noted that some of the less robust associations, which we presumed to be due to smaller sample size, were found between the ADH4 gene and drug dependence only (ie, without comorbid alcohol dependence). This supports the first explanation, although further study of this question with a larger sample of such individuals is warranted. This explanation may help to shed light on the pharmacological mechanism by which disulfiram, an ALDH blocker that is thought to exert its treatment effect in alcohol dependence by interfering with acetaldehyde metabolism, decreases the relapse rate among cocaine-dependent individuals, even in the absence of comorbid alcohol dependence (Carroll et al, 1993, 1998, 2000, 2004; George et al, 2000; Petrakis et al, 2000).

In summary, findings from this study show that the seven markers at $A D H 4$ are associated with alcohol dependence and drug dependence in EAs. Our results suggest that, of these SNPs, the functional variant SNP2 (rs1042363) at exon 9 and SNP6 (rs1800759) in the promoter region are the most important and may affect the risk for both alcohol dependence and drug dependence. Other susceptibility SNPs are in strong LD with SNP2 or SNP6. If the disease risk locus is not SNP2 or SNP6 itself, the putative disease 
risk locus may reside within an LD block containing SNP2 or SNP6 at ADH4 gene.

\section{ACKNOWLEDGEMENTS}

This work was supported in part by funds from the US Department of Veterans Affairs (the VA Medical Research Program, the VA Connecticut-Massachusetts Mental Illness Research, Education and Clinical Center (MIRECC), and the VA Research Enhancement Award Program (REAP) research center), NIH Grants K02-MH01387, R01-DA12849, R01-DA12690, K24-DA15105, R01-AA11330, P50-AA12870, K08-13732, K24-AA13736, and M01-RR06192 (University of Connecticut General Clinical Research Center), a National Alliance for Research on Schizophrenia and Depression (NARSAD) Young Investigator Award (X Luo), and the NIH Biological Sciences Training Program (BSTP) MH14276 (BZ Yang). Ann Marie Lacobelle and Greg Kay provided excellent technical assistance. Dr Ke Hao (Harvard EPH) kindly provided access to his software. Dr Hongyu Zhao (Yale EPH) provided helpful comments on the manuscript.

\section{REFERENCES}

American Psychiatric Association (1994). Diagnostic and Statistical Manual of Mental Disorders, 4th edn. American Psychiatric Press: Washington, DC.

Anokhina IP, Kogan BM, Man'kovskaia IV, Reshchikova EV, Stanishevskaia AV (1990). The similarity of the pathogenetic mechanisms of alcoholism and drug dependence and the means of searching for agents for treating these diseases. Farmakol Toksikol 53: 4-9 (Russian).

Barrett J, Fry B, Maller J, Daly J (2005). Haploview: analysis and visualization of LD and haplotype maps. Bioinformatics 21: 263-265.

Carroll KM, Fenton LR, Ball SA, Nich C, Frankforter TL, Shi J et al (2004). Efficacy of disulfiram and cognitive behavior therapy in cocaine-dependent outpatients: a randomized placebo-controlled trial. Arch Gen Psychiatry 61: 264-272.

Carroll KM, Nich C, Ball SA, McCance-Katz EF, Frankforter T, Rounsaville BJ (2000). One year follow-up of disulfiram and psychotherapy for cocaine-alcohol abusers: sustained effects of treatment. Addiction 95: 1335-1349.

Carroll KM, Nich C, Ball SA, McCance-Katz EF, Rounsavile BJ (1998). Treatment of cocaine and alcohol dependence with psychotherapy and disulfiram. Addiction 93: 713-727.

Carroll KM, Ziedonis D, O’Malley SS, McCance-Katz E, Gordon L, Rounsaville BJ (1993). Pharmacologic interventions for abusers of alcohol and cocaine: a pilot study of disulfiram versus naltrexone. Am J Addict 2: 77-79.

Cordell HJ (2002). Epistasis: what it means, what it doesn't mean, and statistical methods to detect it in humans. Hum Mol Genet 11: 2463-2468.

Devlin B, Risch N (1995). A comparison of linkage disequilibrium measures for fine scale mapping. Genomics 29: 311-322.

Edenberg HJ, Jerome RE, Li M (1999). Polymorphism of the human alcohol dehydrogenase $4(A D H 4)$ promoter affects gene expression. Pharmacogenetics 9: 25-30.

Feder JN, Gnirke A, Thomas W, Tsuchihashi Z, Ruddy DA, Basava A et al (1996). A novel MHC class I-like gene is mutated in patients with hereditary haemochromatosis. Nat Genet 13: 399-408.

Foroud T, Dick D, Xuei X, Goate A, Porjesz B, Begleiter H et al (2003). Association of SNPs in the ADH gene cluster with alcoholism. Am J Hum Genet 73(5 Suppl): 521 (meeting abstract).

Gabriel SB, Schaffner SF, Nguyen H, Moore JM, Roy J, Blumenstiel $\mathrm{B}$ et al (2002). The structure of haplotype blocks in the human genome. Science 296: 2225-2229.

George TP, Chawarski MC, Pakes J, Carroll KM, Kosten TR, Schottenfeld RS (2000). Disulfiram versus placebo for cocaine dependence in buprenorphine-maintained subjects: a preliminary trial. Biol Psychiatry 47: 1080-1086.

Glatt MM (1967). Problems common to alcoholism and drug dependence. WHO Chron 21: 293-303.

Gossop M (2004). Multiple substance use and multiple dependencies. In: Kranzler HR, Tinsley JA (eds). Dual Diagnosis and Psychiatric Treatment. Marcel Dekker: New York. pp 129-156.

Hao K, Xu X, Laird N, Wang X, Xu X (2004). Power estimation of multiple SNP association test of case-control study and application. Genet Epidemiol 26: 22-30.

Hasin D, Aharonovich E, Liu X, Mamman Z, Matseoane K, Carr L et al (2002). Alcohol and $\mathrm{ADH} 2$ in Israel: Ashkenazis, Sephardics, and recent Russian immigrants. Am J Psychiatry 159: 1432-1434.

Heil SH, Badger GJ, Higgins ST (2001). Alcohol dependence among cocaine-dependent outpatients: demographics, drug use, treatment outcome and other characteristics. J Stud Alcohol 62: $14-22$.

Hoehe MR, Kopke K, Wendel B, Rohde K, Flachmeier C, Kidd KK et al (2000). Sequence variability and candidate gene analysis in complex disease: association of mu opioid receptor gene variation with substance dependence. Hum Mol Genet 19: 2895-2908.

Hoh J, Wille A, Ott J (2001). Trimming, weighting, and grouping SNPs in human case-control association studies. Genome Res 11: 2115-2119.

Jiang R, Dong J, Wang D, Sun FZ (2001). Fine-scale mapping using Hardy-Weinberg disequilibrium. Ann Hum Genet 65: 207-219.

Konishi T, Calvillo M, Leng AS, Feng J, Lee T, Lee H et al (2003). The $\mathrm{ADH}^{*} 2$ and $\mathrm{CYP} 2 \mathrm{E} 1 \mathrm{c} 2$ alleles increase the risk of alcoholism in Mexican American men. Exp Mol Pathol 74: 183-189.

Kreek MJ, Nielsen DA, LaForge KS (2004). Genes associated with addiction: alcoholism, opiate, and cocaine addiction. Neuromol Med 5: 85-108.

Lee WC (2003). Searching for disease-susceptibility loci by testing for Hardy-Weinberg disequilibrium in a gene bank of affected individuals. Am J Epidemiol 158: 397-400.

Li TK, Bosron WF (1987). Distribution and properties of human alcohol dehydrogenase isoenzymes. Ann NY Acad Sci 492: $1-10$.

Li TK, Bosron WF, Dafeldecker WP, Lange LG, Vallee BL (1977). Isolation of PI-alcohol dehydrogenase of human liver: is it a determinant of alcoholism? Proc Nat Acad Sci USA 74: 43784381.

Liu K, Muse S (2004). PowerMarker: new genetic data analysis software. Version 3.0. Free program distributed by the author over the internet from http://www.powermarker.net. Accessed October 1, 2004.

Long JC, Knowler WC, Hanson RL, Robin RW, Urbanek M, Moore $\mathrm{E}$ et al (1998). Evidence for genetic linkage to alcohol dependence on chromosomes 4 and 11 from an autosome-wide scan in an American Indian population. Am J Med Genet 81: $216-221$.

Luo X, Kranzler HR, Zuo L, Yang BZ, Lappalainen J, Gelernter J $\mathrm{ADH} 4$ gene variation is associated with alcohol dependence and drug dependence in European Americans: results from family-controlled and population-structured association studies. Pharmacogenet Genomics (in press).

Luo X, Kranzler HR, Zhao H, Gelernter J (2003). Haplotypes at the OPRM1 locus are associated with susceptibility to substance 
dependence in European-Americans. Am J Med Genet (Neuropsychiatric Genet) 120B: 97-108.

Luo X, Kranzler HR, Zuo L, Wang S, Blumberg HP, Gelernter J (2005). CHRM2 gene predisposes to alcohol, drug dependence and affective disorder: results from an extended population structured association study. Hum Mol Genet 14: 2421-2434.

Muramatsu T, Wang ZC, Fang YR, Hu KB, Yan H, Yamada K et al (1995). Alcohol and aldehyde dehydrogenase genotypes and drinking behavior of Chinese living in Shanghai. Hum Genet 96: 151-154.

Nielsen DM, Ehm MG, Weir BS (1998). Detecting marker-disease association by testing for Hardy-Weinberg disequilibrium at a marker locus. Am J Hum Genet 63: 1531-1540.

Niu T, Qin ZD, Xu X, Liu JS (2002). Bayesian haplotype inference for multiple linked single-nucleotide polymorphisms. Am J Hum Genet 70: 157-169.

O’Neill J, Cardenas VA, Meyerhoff DJ (2001). Separate and interactive effects of cocaine and alcohol dependence on brain structures and metabolites: quantitative MRI and proton MR spectroscopic imaging. Addict Biol 6: 347-361.

Petrakis IL, Carroll KM, Nich C, Gordon LT, McCance-Katz EF, Frankforter $\mathrm{T}$ et al (2000). Disulfiram treatment for cocaine dependence in methadone maintained opioid addicts. Addiction 95: 219-228.

Reich T, Edenberg HJ, Goate A, Williams JT, Rice JP, Van Eerdewegh $\mathrm{P}$ et al (1998). Genome-wide search for genes affecting the risk for alcohol dependence. Am J Med Genet 81: 207-221.

Robinson JE, Heaton RK, O’Malley SS (1999). Neuropsychological functioning in cocaine abusers with and without alcohol dependence. J Int Neuropsychol Soc 5: 10-19.

Saccone NL, Kwon JM, Corbett J, Goate A, Rochberg N, Edenberg $\mathrm{HJ}$ et al (2000). A genome screen of maximum number of drinks as an alcoholism phenotype. Am J Med Genet 96: 632-637.
Sasieni PD (1997). From genotypes to genes: doubling the sample size. Biometrics 53: 1253-1261.

Schinka JA, Town T, Abdullah L, Crawford FC, Ordorica PI, Francis E et al (2002). A functional polymorphism within the mu-opioid receptor gene and risk for abuse of alcohol and other substances. Mol Psychiatry 7: 224-228.

Shi MM, Myrand SP, Bleavins MR, de la Iglesia FA (1999). High throughput genotyping for the detection of a single nucleotide polymorphism in $\mathrm{NAD}(\mathrm{P}) \mathrm{H}$ quinone oxidoreductase (DT diaphorase) using TaqMan probes. Mol Pathol 52: 295-299.

Stephens M, Donnelly P (2003). A comparison of bayesian methods for haplotype reconstruction from population genotype data. Am J Hum Genet 73: 1162-1169.

Stephens M, Smith NJ, Donnelly P (2001). A new statistical method for haplotype reconstruction from population data. Am J Hum Genet 68: 978-989.

Szeto CY, Tang NL, Lee DT, Stadlin A (2001). Association between $\mathrm{mu}$ opioid receptor gene polymorphisms and Chinese heroin addicts. Neuroreport 6: 1103-1106.

Thomasson HR, Crabb DW, Edenberg HJ, Li TK, Hwu HG, Chen $\mathrm{CC}$ et al (1994). Low frequency of the $\mathrm{ADH} 2 * 2$ allele among Atayal natives of Taiwan with alcohol use disorders. Alcohol Clin Exp Res 18: 640-643.

Williams JT, Begleiter H, Porjesz B, Edenberg HJ, Foroud T, Reich $\mathrm{T}$ et al (1999). Joint multipoint linkage analysis of multivariate qualitative and quantitative traits. II. Alcoholism and eventrelated potentials. Am J Hum Genet 65: 1148-1160.

Wittke-Thompson JK, Pluzhnikov A, Cox NJ (2005). Rational inferences about departures from Hardy-Weinberg equilibrium. Am J Hum Genet 76: 967-986.

Zinn-Justin A, Abel L (1999). Genome search for alcohol dependence using the weighted pairwise correlation linkage method: interesting findings on chromosome 4. Genet Epidemiol 17(Suppl 1): S421-S426. 